\title{
Ultra-Low-Power Intelligent Acoustic Sensing using Cochlea-Inspired Feature Extraction and DNN Classification
}

\author{
Minhao Yang ${ }^{1}$, Shih-Chii Liu ${ }^{2}$, Mingoo Seok ${ }^{3}$, Christian Enz $^{1}$ \\ ${ }^{1}$ Institute of Microengineering, EPFL, Neuchatel 2000, Switzerland \\ ${ }^{2}$ Institute of Neuroinformatics, UZH/ETH Zurich, Zurich 8057, Switzerland \\ ${ }^{3}$ Department of Electrical Engineering, Columbia University, NY 10027, USA \\ * Email: minhao.yang@epfl.ch
}

\begin{abstract}
We present our recent progress in ultra-low-power intelligent acoustic sensing that harnesses the high power and energy efficiency of cochlea-like analog feature extraction and binarized neural network classification. Compared with conventional methods including the fast Fourier transform-based feature extraction plus neural network classification, and the more aggressive approach based on end-to-end neural network models, the analog filter bank-based handcrafted feature extraction inspired by mammalian cochlea has the promise of achieving the minimum power consumption for many existing and emerging always-on audio applications. System considerations and circuit techniques that are used to achieve the high power efficiency will be presented and comparison with some state-of-the-art works, and future directions will be discussed.
\end{abstract}

\section{Introduction}

Ultra-low-power (ULP) intelligent acoustic interfaces are finding applications in wearable electronics like wireless earbuds, smart watches and mobile phones, and may in the future enable truly immersive ambient intelligence that can interact with people through natural conversations. Among all the intelligent functionalities in audio processing systems, voice activity detection (VAD) that distinguishes speech from background noise is perhaps the most widely used given that it can serve as a monitor [1,2] that wakes up more energy-consuming building blocks, such as keyword spotting (KWS), speaker identification (SID), automatic speech recognition (ASR) etc., when speech is detected. As an example, one such system is illustrated in Fig. 1. The wake-up signal from the VAD can be used for power-gating control of the blocks in the dashed square, and its average duty cycle and the other blocks' energy efficiency determine the upper bound of the VAD's power budget. For example, one wants to use voice commands to unlock the screen of a smart watch, and this requires a system that consists of VAD and SID. If the SID consumes $100 \mu \mathrm{W}$ and its duty cycle is $10 \%$, the VAD should consumes not more than $5 \mu \mathrm{W}$ to impose a $50 \%$ power overhead.

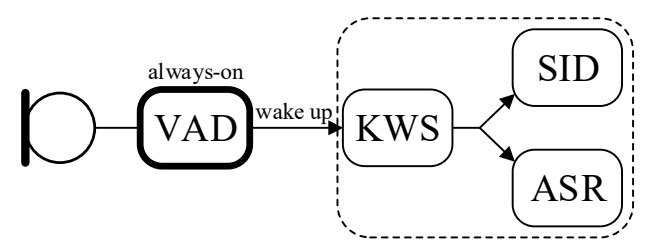

Fig. 1. An audio processing system with the always-on VAD block that wakes up the more energy-consuming blocks including KWS, SID, and ASR, when speech is detected.

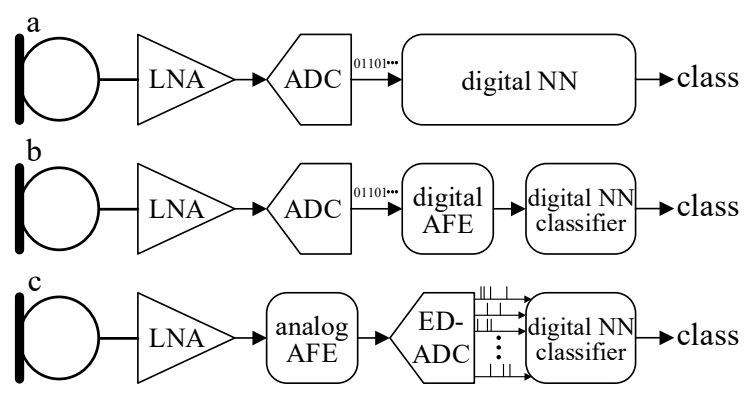

Fig. 2. Three different intelligent acoustic interface architectures: digital-intensive architectures (a) that relies on digital NN to perform AFE and classification, (b) that relies on a dedicated DSP for AFE and digital NN for classification; (c) analog-intensive architecture that relies on analog signal processing for cochlea-inspired AFE and digital $\mathrm{NN}$ for classification.

Inference sensing tasks like VAD can be functionally divided into two parts, acoustic feature extraction (AFE) and classification. With the rise of deep learning [3], the AFE that previous relies on domain-specific expertise may now be replaced by deep neural network (DNN) as illustrated in Fig. 2(a) where the feature extraction and classification are unified in one neural network architecture, the same as in many vision tasks like image recognition and object detection. While DNN-based feature extraction can largely benefit the inference accuracy, it is at the expense of significantly lower energy efficiency when compared with the handcrafted approaches like generating the histogram of oriented gradients (HOG) 
features [4]. Similarly, for audio signals, we can expect the NN-based AFE has the problem of high power consumption given the high input dimensionality, and yet this approach does not necessarily guarantee improvement in inference performance, as is shown for ASR in [5]. Without any obvious advantage, the NN-based AFE does not appear attractive compared to the conventional handcrafted AFE.

One type of the most widely used handcrafted acoustic features for a variety of audio inference tasks is the mel-frequency cepstral coefficients (MFCC) [6]. It mainly involves discrete Fourier transform (DFT), log energy computation of different mel-scaled bandpass frequency bands, and discrete cosine transform. These signal processing steps are commonly implemented in digital circuits $[1,2]$ as represented by the digital AFE block in Fig. 2(b) and are not friendly to analog implementations. The state-of-the-art VAD using an architecture of digital AFE plus fixed-point DNN consumes $22 \mu \mathrm{W}$ [2], limiting the battery life in always-on sensing. It has been theoretically established that analog circuits are more energy efficient when the signal-to-noise ratio (SNR) requirement in a signal processing pipeline is not high $[7,8]$. In the field of neuromorphic engineering started in the late 1980 s, this principle was extensively exploited in building electronic sensory systems like artificial cochlea [9-12] and retina [13], and biologically plausible spiking neural networks (SNN) [14]. Particularly in silicon cochlea, the AFE is performed in analog domain, and the extracted features are encoded into parallel digital events by event-driven analog-to-digital converters (ED-ADC) for further processing, as illustrated in Fig. 2(c). This approach is the focus of this review. Its potential of ULP performance becomes increasingly attractive in the era of Artificial Intelligence meeting Internet of Things (AIoT).

\section{Silicon Cochlea for Acoustic Feature Extraction}

One key functionality in analog AFE is the massively parallel bandpass filtering with nearly geometrical central frequency scaling, similar to the mammalian cochlea transfer functions across the basilar membrane. It is the first processing step in extracting and estimating the energy content of each frequency band. Another key functional block for input-activity-dependent sparse coding is the ED-ADC. With new circuit techniques for implementing these two essential functions, our attempt of building an ULP AFE system resulted in a $55 \mu \mathrm{W}$ binaural silicon cochlea with $64 \times 2$ channels, which for the first time enables well intelligible input speech reconstruction using the asynchronous output events through simple signal processing $[15,16]$.

The main signal processing blocks in one channel are shown in Fig. 3. The lowpass filter (LPF) and the programmable gain amplifier (PGA) together form the

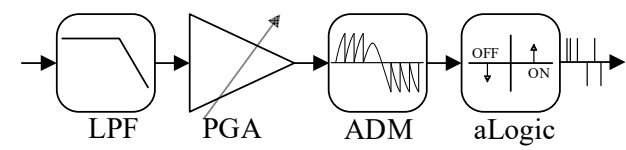

Fig. 3. Signal processing chain in a monaural channel of the silicon cochlea.
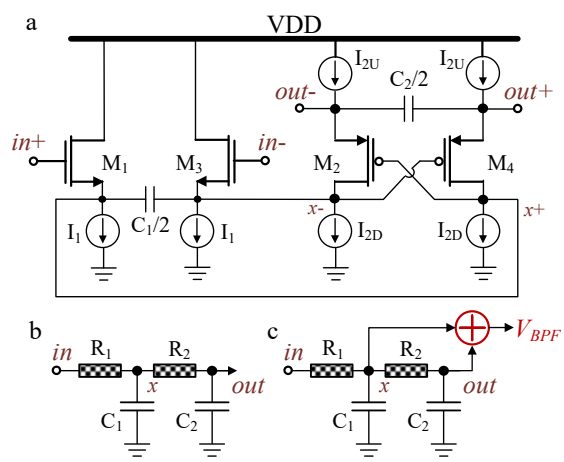

Fig. 4. (a) Circuit schematic of an SSB-LPF; (b) its RC-equivalent circuit; (c) an SSB-BPF derived from an SSB-LPF.

bandpass filter (BPF). The asynchronous delta modulator (ADM) [17] serves the function of an ED-ADC. The asynchronous logic (aLogic) sends the generated events off-chip through address event representation (AER) circuits [18]. Here we describe the BPF design in detail.

The BPF is devised from a source-follower-based (SSB) LPF, which was initially intended for wireless transceiver applications with high power efficiency $[19,20]$. The transistor-level SSB-LPF and its RC-equivalent circuits are shown in Fig. 4(a) and 4(b), respectively. The value of $R_{1}$ and $R_{2}$ is the reciprocal of the transconductance of $M_{1}$ and $M_{2}$, i.e. $g_{m 1}$ and $g_{m 2}$, respectively. Because of the cross-coupled pFETs, $\mathrm{R}_{2}$ has a negative resistance, and it is crucial for obtaining a quality factor $\mathrm{Q}$ larger than 0.5 . By observing the transfer function at nodes $x$ and out, it is easy to find out that a $2^{\text {nd }}$-order BPF transfer function can be obtained by summing the voltages:

$$
H_{B P F}(s)=\frac{s \cdot \frac{\mathrm{C}_{2}}{g_{m 2}}}{s^{2} \cdot \frac{\mathrm{C}_{1} \mathrm{C}_{2}}{g_{m 1} g_{m 2}}+s \cdot\left(\frac{\mathrm{C}_{1}}{g_{m 1}}+\frac{\mathrm{C}_{2}}{g_{m 2}}-\frac{\mathrm{C}_{2}}{g_{m 1}}\right)+1}
$$

The summation is achieved through the PGA with capacitive feedback. Its input capacitors are at the same time the loading capacitors $\mathrm{C}_{1}$ and $\mathrm{C}_{2}$ of the BPF.

A high Q may be beneficial for some difficult cognitive tasks, as is suggested by the high tuning sharpness observed in mammalian cochlea. However, a high $Q$ in analog circuits can be very susceptible to device mismatch. By performing parameter sensitivity analysis, at 


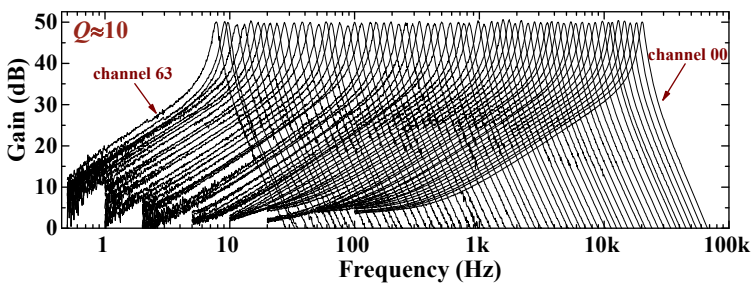

Fig. 5. Measured transfer functions of the BPF bank at $\mathrm{Q}=10$.
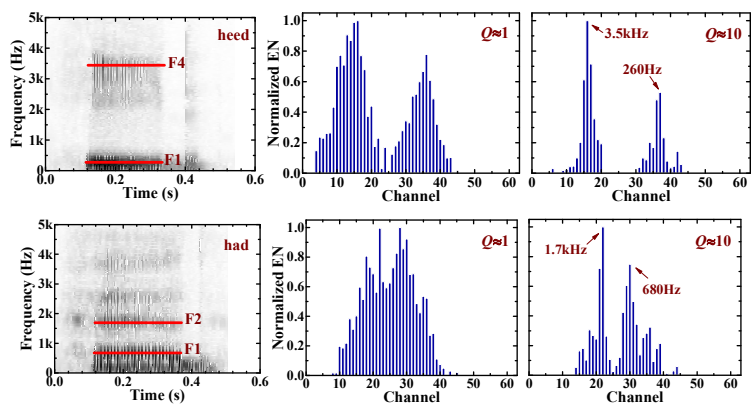

Fig. 6. Spectrograms of utterances "heed" and "had" with most-energy-significant vowel formants labeled; measured event histograms with the two utterances as the input at $\mathrm{Q}=1$ and $\mathrm{Q}=10$.

$\mathrm{Q}=10$, we see that in the extreme case of choosing $\mathrm{C}_{1}=\mathrm{C}_{2}$, $1 \%$ mismatch between $\mathrm{I}_{2 \mathrm{U}}$ and $\mathrm{I}_{2 \mathrm{D}}$ results in $41 \%$ deviation of the central frequency $\mathrm{f}_{0}$. In the other extreme case of choosing $\mathrm{g}_{\mathrm{m} 1}=\mathrm{g}_{\mathrm{m} 2}$, we see that $1 \%$ mismatch between $\mathrm{g}_{\mathrm{m} 1}$ and $\mathrm{g}_{\mathrm{m} 2}$ results in $99.5 \%$ variation of $\mathrm{Q}$. As a tradeoff between $\mathrm{f}_{0}$ and $\mathrm{Q}$ sensitivities, we chose $\mathrm{C}_{2} / \mathrm{C}_{1}=26 / 15$. At $\mathrm{Q}=10$, this gives an $\mathrm{f}_{0}$ deviation of $1 \%$ with a $1 \%$ mismatch between $\mathrm{I}_{2 \mathrm{U}}$ and $\mathrm{I}_{2 \mathrm{D}}$, and $8 \%$ deviation of $\mathrm{Q}$ with a $1 \%$ mismatch between $\mathrm{g}_{\mathrm{m} 1}$ and $\mathrm{g}_{\mathrm{m} 2}$.

Fig. 5 shows the measured transfer functions of the BPF bank at $\mathrm{Q}=10$. The central frequencies monotonically scale from about $8 \mathrm{~Hz}$ to $20 \mathrm{kHz}$ with a scaling ratio of 1.13. Fig. 6 shows the application of the silicon cochlea in vowel discrimination. It is evident that a higher $\mathrm{Q}$ facilitates the identification of the characteristic formants of different vowels.

\section{Ultra-Low-Power Voice Activity Detection}

To apply the event-driven AFE in real-life always-on inference sensing, we chose VAD as the starting point. The system architecture of the VAD is shown in Fig. 7 $[21,22]$. The AFE and the classification based on binarized neural network (BNN) [23] uses analog and digital circuits, respectively. For a higher BNN energy efficiency, mixed-signal in-memory computing could have been used [24,25]. In order to focus on the challenge of the PVT variation of the analog AFE, we chose to implement a conventional Von Neumann digital classifier.

Two main differences in the AFE processing chain com-

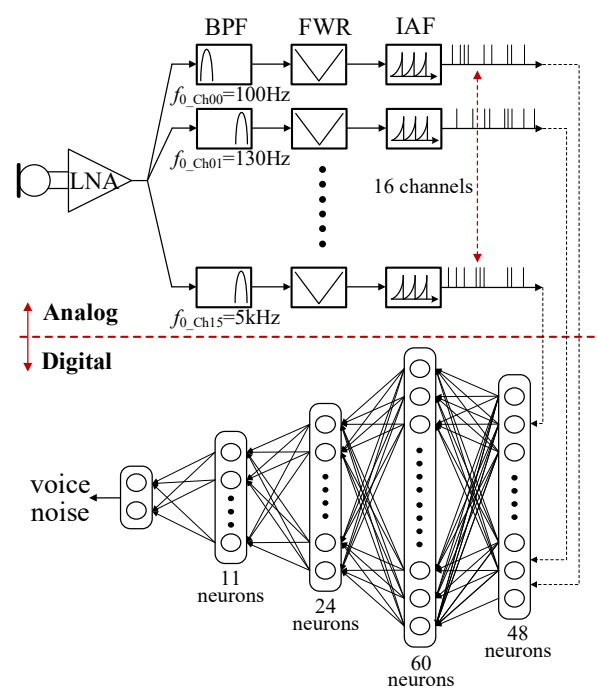

Fig. 7. VAD system architecture using cochlea-inspired $\mathrm{AFE}$ and $\mathrm{BNN}$ classification.

pared to the prior work are the added full-wave rectification (FWR) and the integrate-and-fire (IAF) event encoding. These two steps are necessary in constructing the cochleagram representation that is widely used as the features in computational auditory scene analysis [26]. The AFE can be expressed as:

$$
\frac{1}{\mathrm{C}_{\mathrm{int}}} \int_{t_{j}}^{t_{j+1}}\left|f_{v \rightarrow i}\left(v_{o B F_{-} k}(t)\right)\right| \mathrm{d} t=\mathrm{V}_{\text {refdn }}
$$

where $t_{j}$ is the timestamp of the $\mathrm{j}^{\mathrm{t}}$ event, $v_{O B P F_{-} k}$ is the BPF output voltage in channel $\mathrm{k}, f_{v \rightarrow i}$ is the function of voltage to current conversion in FWR, and $\mathrm{C}_{\text {int }}$ and $\mathrm{V}_{\text {refdn }}$ is the integration capacitance and the threshold voltage of the IAF, respectively. The accumulated number of events in each channel in a $25-\mathrm{ms}$ frame is used as the input feature for the BNN, which is the estimation of the area under the rectified waveform.

The digital BNN's input dimension is expanded from 16 AFE channels to 48 by incorporating contextual information using neighboring frames: to classify frame i, frame i-3 and i+3 are also used. Empirically this has been shown to improve the classification performance [26]. The light BNN has 3 hidden layers and the output layer has two neurons, with one's activation larger than the other indicating voice, and noise otherwise.

The $1 \mu \mathrm{W}$ power consumption of the VAD at a throughput of 100 class/s is achieved by extensively using proposed and existing ULP techniques. Some of the examples are: in the LNA, inverter-based input stage with a single tail current enabled by a scaled replica of the inverter, and positive feedback to extend the bandwidth with reduced bias in the output stage; in the BPF with $\mathrm{Q} \approx 2$, super-source-follower-based topology without any amplifier; in the IAF, a slow comparator with its delay 


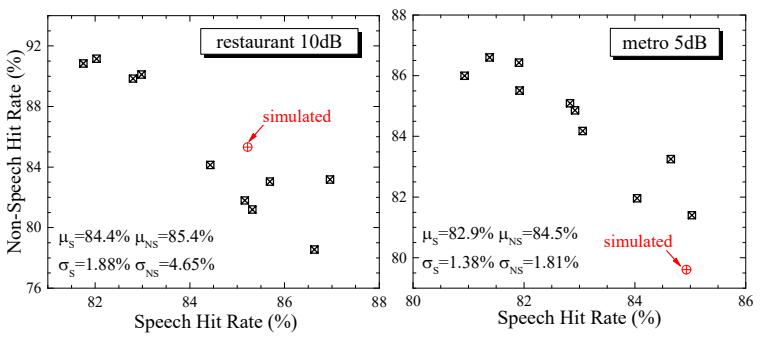

Fig. 8. The measured speech/non-speech hit rate using the $10 \mathrm{~dB}$ noisy speech with restaurant noise, and the $5 \mathrm{~dB}$ noisy speech with metro noise. The same weights trained off-line are used for the MLP in all 10 test chips.

considered in the feature extraction modeling; in the digital BNN, reduced supply voltage for both logic and memory, and clock gating.

One of the main problems of using analog circuits for AFE is the performance variation. Previously, researchers have been relying on the adapting capability of machine learning (ML) models to account for the large AFE variation after chip fabrication [27,28]. However, this is impractical in mass production because of the costly characterization and ML model training for every chip. To study the impact of circuit-induced feature variation on classification performance and to guide the circuit design, we built a dedicated AFE software model that accurately emulates the transistor-level hardware system with significantly improved simulation speed compared to using tools like Cadence Virtuoso. During chip testing, the BNN parameters were obtained by training the BNN model using the features from the software AFE, and loaded to all 10 tested chips. As shown in Fig. 8, the mean speech and non-speech hit rate, $\mu_{\mathrm{S}}$ and $\mu_{\mathrm{NS}}$, and the corresponding 1- $\sigma$ variation, $\sigma_{S}$ and $\sigma_{N S}$, are given in the plot. Overall, the software simulation result using the customized AFE software model is a good prediction of the measured system classification performance. It is worth investigating in future if the hit rate variation can be reduced by considering the feature variation as some form of noise during DNN training.

\section{Discussion and Future Works}

One recent work on VAD reaches a record low $142 \mathrm{nW}$ [29], but the throughput is only 2class/s. The key technique of using frequency-scanning mixer to extract the energy in each frequency band is at the expense of long feature extraction time for each frame. The $0.5 \mathrm{~s}$ latency implies that it cannot be used in many practical applications that are delay sensitive. For example, in hearing aids evaluation, $25 \mathrm{~ms}$ to $30 \mathrm{~ms}$ delay is already deemed as disturbing [30]. Comparing the energy efficiency, our VAD's is $10^{8} \mathrm{class} / \mathrm{J}$, and [29]'s is $1.4 \times 10^{7} \mathrm{class} / \mathrm{J}$.

To further push the limit of energy efficiency, we are currently working on a $\sim 100$ nW VAD with the same throughput of 100class/s. The bottleneck of the AFE in our previous work is the FWR which consumes more than $50 \%$ of the AFE power. Through hardware/software co-design, we find out a way of significantly reducing the power needed in rectification while maintaining the same level of speech/non-speech hit rate. We are also exploring using this new cochlea inspired AFE for KWS. The details of the on-going work will be published soon.

\section{References}

[1] A. Raychowdhury, C. Tokunaga, W. Beltman, M. Deisher, J. W. Tschanz and V. De, IEEE JSSC, 48, p.1963 (2013).

[2] M. Price, J. Glass and A. P. Chandrakasan, IEEE JSSC, 53, p.66 (2018).

[3] Y. LeCun, Y. Bengio, and Hinton, Nature, 521, p. 436 (2015)

[4] A. Suleiman, Y.-H. Chen, J. Emer, and V. Sze, IEEE ISCAS, p. 1 (2017).

[5] Y. Hoshen, R. J. Weiss, and K. W. Wilson, IEEE ICASSP, p. 4624 (2015).

[6] S. B. Davis, and P. Mermelstein, IEEE TASSP, 28, p. 357 (1980).

[7] B. J. Hosticka, Proc. IEEE, 73, p. 25 (1985).

[8] R. Sarpeshkar, Neural Computation, 10, p. 1601 (1998).

[9] R. F. Lyon, and C. Mead, IEEE TASSP, 36, p. 1119 (1988).

[10]A. Schaik, E. Fragniere, and E. Vittoz, NIPS, p. 671 (1995)

[11]V. Chan, S.-C. Liu, and A. Schaik, IEEE TCAS-I, 54, p. 48 (2007).

[12]S.-C. Liu, A. Schaik, B. A. Minch, and T. Delbruck, IEEE TBioCAS, 8, p. 453 (2014).

[13]K. A. Boahen, IEEE Micro, 16, p. 30 (1996)

[14]G. Indiveri, E. Chicca, and R. Douglas, IEEE TNN, 17, p. 211 (2006).

[15]M. Yang, C.-H. Chien, T. Delbruck, and S.-C. Liu, IEEE ISSCC, p. 388 (2016).

[16]M. Yang, C.-H. Chien, T. Delbruck, and S.-C. Liu, IEEE JSSC, 51, p. 2554 (2016).

[17]H. Inose, T. Aoki, and K. Watanabe, IET EL, 2, p. 95 (1966).

[18]K. A. Boahen, IEEE TCAS-I, 51, p. 1269 (2004).

[19]S. D'Amico, M. Conta, and A. Baschirotto, IEEE JSSC, 41, p. 2713 (2006)

[20]S. D'Amico, M. Matteis, and A. Baschirotto, IEEE ISSCC, p. 72 (2008).

[21]M. Yang, C.-H. Yeh, Y. Zhou, J. P. Cerqueira, A. A. Lazar, and M. Seok, IEEE ISSCC, p. 346 (2018).

[22]M. Yang, C.-H. Yeh, Y. Zhou, J. P. Cerqueira, A. A. Lazar, and M. Seok, IEEE JSSC, 54, p. 1764 (2019).

[23]I. Hubara, M. Courbariaux, D. Soudry, R. El-Yaniv, and Y. Bengio, NIPS, p. 4107 (2016).

[24]D. Bankman, L. Yang, B. Moons, M. Verhelst, and B. Murmann, IEEE JSSC, 54, p. 158 (2019).

[25]A. Biswas, and A. P. Chandrakasan, IEEE JSSC, 54, p. 217 (2019).

[26]X.-L. Zhang, and D. Wang, IEEE/ACM TASLP, 24, p. 252 (2016).

[27]J. Zhang, L. Huang, Z. Wang, and N. Verma, IEEE CICC, p. 1 (2015).

[28]K. M. H. Badami, S. Lauwereins, W. Meert, and M. Verhelst, IEEE JSSC, 51, p. 291 (2016).

[29]M. Cho, S. Oh, Z. Shi, J. Lim, Y. Kim, S. Jeong, Y. Chen, D. Blaauw, H.-S Kim, and D. Sylvester, IEEE ISSCC, p. 278 (2019).

[30]M. A. Stone, and B. C. J. Moore, Ear and Hearing, 23, p. 325 (2002). 